\title{
From 3D Model Data to SEmantics
}

\author{
My Abdellah Kassimi ${ }^{1}$ and Omar El beqqali ${ }^{2}$ \\ ${ }^{1,2}$ Sidi Mohamed Ben Abdellah University/ LIIAN, GRMS2I, Fez, Morocco \\ labdellahkassimi@yahoo.fr \\ 2 omarelbeqqaliegmail.com
}

\begin{abstract}
The semantic-based 3D models retrieval systems have become necessary since the increase of 3D models databases. In this paper, we propose a new method for the mapping problem between $3 D$ model data and semantic data involved in semantic based retrieval for $3 D$ models given by polygonal meshes. First, we focused on extracting invariant descriptors from the $3 D$ models and analyzing them to efficient semantic annotation and to improve the retrieval accuracy. Selected shape descriptors provide a set of terms commonly used to describe visually a set of objects using linguistic terms and are used as semantic concept to label 3D model. Second, spatial relationship representing directional, topological and distance relationships are used to derive other high-level semantic features and to avoid the problem of automatic 3D model annotation. Based on the resulting semantic annotation and spatial concepts, an ontology for 3D model retrieval is constructed and other concepts can be inferred. This ontology is used to find similar $3 D$ models for a given query model. We adopted the query by semantic example approach, in which the annotation is performed mostly automatically. The proposed method is implemented in our $3 D$ search engine (SB3DMR), tested using the Princeton Shape Benchmark Database.
\end{abstract}

\section{KEYWORDS}

$3 D$ model, classification, 3D retrieval, semantic, ontology, annotation, shape index

\section{INTRODUCTION}

In recent years, 3D object databases have grown in both size and importance and 3D model is becoming more commonly available on the internet and domain-specific databases due to the fast advancement of tools for acquisition and storage of 3D models. Therefore, efficient content based 3D model retrieval (CB3DMR) systems become increasingly important. These systems allow users to find similar 3D shapes for a given query shape and database of 3D models. On the other hand, these systems are traditionally performed using Keywords and the geometry. 3D model semantic retrieval is an important and challenging task in the field of (CB3DMR). However, there are several systems that are manually annotated 3D models to include semantic information. It is thus necessary to develop content based 3D model retrieval systems for 3D models using their semantic content automatically annotated. Many researchers are encouraged to develop semantic based 3D model retrieval systems for 3D models [3].In this context, the challenge is to provide an efficient high-level semantic interpreting for computing $3 \mathrm{D}$ model similarity [4].

The main problem of semantic based 3D shape systems is extracting model's shape features with good properties and representing visual concepts. In this paper, shape descriptors are analyzed to represent visual concepts and to construct the Ontology-based 3D model retrieval system. We adopted the query by semantic example approach, in which the annotation is performed automatically. Shape descriptors are divided into four categories: measures, size, shape indexes, and moments of distribution descriptors. First, measures are extracted from each 3D model, from convex hull and bounding box in the database and we compute invariant 3D shape indexes for characterizing 3D shapes. Each shape index describes an entire 3D shape.

DOI : $10.5121 /$ ijcsit.2011.3601 
With one single shape index is insufficient for identifying different properties. Then, the combination of shape indexes is often required. Four independent categories or properties of shape indexes may be considered to be of particular importance: Sphericity, Convexity, Compactness and Elongation. Each property measures a different aspect and can vary without affecting the other properties [13].

Sphericity and Compactness are represented by independent Shape indexes to reflect form and angularity at the corners on shape and to be more efficient discriminators than simple measures. Elongation is used to determine whether a 3D model has the flat or the elongated shape. Third category of shape indexes, calculated from convex hull, captures information about global convexity and measure the surface roughness. Each Shape index is normalized between 0 and 1 and has the advantage of being invariant to different transformations [14]. Shape indexes in each category are very easy to compute and efficient for broad retrieval of 3D shapes.

Shape descriptors only cannot represent 3D model semantics. Spatial relationships that are usually defined according to the location of the measure in the 3D model, can overcome these limitations by describing spatial relationships among entities. Four points from convex hull are considered. During this process, moments of distribution descriptors are calculated.

The second stage is to describe 3D model with semantic concepts. We used shape indexes that provide a set of terms commonly used to describe visually a set of objects using linguistic terms [15]. These terms are accurately quantified and are used as semantic concept to build our ontology. Therefore, a learning method is applied to the shape index category to label 3D model and to construct the visual terms ontology. For example, to label 3D model as "convex" we compute convexity shape indexes using different methods and k-mean algorithm is applied to learn the statistical properties of these shape indexes and to agglomerate clusters. After the labelling process, the labels and spatial concepts of the model are used in Ontology-Based 3D Model Retrieval to describe model information for interpreting its semantic content.

\section{RELATED WORK}

In recent years, several methods are proposed in the literature to describe 3D models. A survey of Content-based 3D model retrieval (CB3DMR) can be found in $[1,2]$. In this paper, we present survey on the CB3DMR methods with high-level semantics. These methods are generally implemented including three categories to reduce the semantic gap: (1) using machine learning methods to associate low-level features with high- level concepts, (2) introducing relevance feedback that is mostly combined with machine learning methods and (3) using ontology to define semantic concepts. In several works, the three methods are combined.

The work presented in [29] introduces the classification and retrieval 3D model by integrating shape features and semantic information. The paper proposes a new type of shape feature based on 2D views and use Gaussian processes as supervised learning to mode the mapping from lowlevel features to query concepts. In the paper [30], author explores a new framework for 3D model retrieval based on an off-line learning of the most salient features of the shapes. The proposed approach uses a large set of features, which can be heterogeneous, to capture the highlevel semantic concepts of different shape classes. Hou and al, in [10] Support Vector Machine (SVM) is used to cluster 3D models with respect to semantic information to organizing a database of shapes. Neural networks and SVM as learning methods are used in [9] for learning the concept.

In order to reduce the semantic gap, authors in [4, 5, 16, 25 and 32] introduce relevance feedback processing combined with various feature representations for 3D model retrieval. Relevance feedback is effective in reducing the semantic gap and it is an interactive scheme that requires the user to label 3D models as relevant or irrelevant [31]. Authors in [5] present a relevance feedback algorithm based on machine learning techniques. This algorithm is 
implemented in 3D shape retrieval system based on 3D Zernike descriptors and allows the user to mark the result as relevant or not to provide the degree of relevance. In paper [4] the author proposes a 3D model retrieval algorithm that is based on the manifold ranking algorithm to use a relevance-feedback framework. The method embodies feature distribution and multiple semantic classes to learn long-lived semantics. C. Zhang in [16] includes relevance feedback in content-based 3D models retrieval system combined with machine learning method. The hidden annotation is incorporated into the system with a set of region-based features and the active learning is used to improve the annotation efficiency. The algorithm proposed in [32] for 3D model retrieval combines SVM technique with active learning mechanism to learn from the most informative objects. The user marks these objects to separate the relevant models from irrelevant ones. Compared with others relevance feedback schemes, the authors obtain maximum information learning from the most informative samples.

The main objective of the Network of Excellence AIM@SHAPE [33] is to capture the implicit semantics of digital shapes and ontologies can be used to encode, store and formalize the domain knowledge. In this context and to add semantics to geometry, Marios in [12] analyzes the 3D shape and can extract and combine knowledge and implicit information coded in the geometry of the digital content object and its sub-parties (volume, surface ...), then it allows the segmentation of 3D shapes based on semantics. Including the spatial relation ontology, author in [15] introduces visual concept ontology as a guideline to describe the specific knowledge of an application domain. To create the link between low-level features and the concept of queries, author in [7] uses machine learning methods and the high-level ontology to define the concepts of 3D objects [8]. The SCULPTEUR system developed in [6] uses class labels stored in domain ontology and a classifier agent automatically creates the link between 3D shape feature vectors and appropriate class labels. This method provides a potential training set for automatic classification. In the paper [11], author explores an ontology and SWRL-based 3D model retrieval system Onto3D. This ontology is used to infer 3D models semantic property through the rule engine and to retrieve the target models by request engine effectively to improve the retrieval accuracy.

The main objective of this paper is to propose a new method in extracting semantic feature for 3D Model that can get high-level semantic knowledge representation automatically from lowlevel feature. It is the most important problem in content based 3D model retrieval. We used a learning method to the shape index category to label 3D model and, based on the resulting semantic annotation, spatial concepts are added to build the ontology-based 3D model retrieval using the web ontology language.

\section{PRE-PROCESSING}

To represent the 3D models, polygonal mesh is the most used. The main difficulty is that $3 \mathrm{D}$ models have the arbitrary topologies and are not normalized. In the pre-processing step, the challenge in computing an ideal shape feature is to remain unaffected by scale, rotation and translation transformation. Basically, there are two ways to address this challenge: 1) Develop a transformation-invariant descriptor or 2) Normalization. Using the first approach, we can extract transformation invariant information from the shape index. Consider, for example the case that we have a model represented by a triangular mesh, if we want to make the shape index "elongation" invariant to rotation, radius and main axis measures are used. Advantages of the shape indexes [14] are 1) they can be computed from measures and providing numerical information about the shape of objects, 2) they are invariants to translation, and rotation transformation, and 3) they are dimensionless. However, measures without dimension are considered shape indexes. 
Normalization techniques obtain invariance by computing Shape indexes relative to a global reference frame presented by the object centroid. From the triangular mesh the object centroid is calculated as follows:

$$
\left(\begin{array}{l}
m_{x} \\
m_{y} \\
m_{z}
\end{array}\right)=\sum_{i=1}^{N}\left(\begin{array}{l}
x_{i} \\
y_{i} \\
z_{i}
\end{array}\right) / N
$$

Where $\left(\mathrm{m}_{\mathrm{x}}, \mathrm{m}_{\mathrm{y}}, \mathrm{m}_{\mathrm{z}}\right)$ is the coordinates of the object center, $\left(\mathrm{x}_{\mathrm{i}}, \mathrm{y}_{\mathrm{i}}, \mathrm{z}_{\mathrm{i}}\right)$ is the coordinates of the object vertex and $\mathrm{N}$ is the number of the vertex which constitutes the object. Using this equation (1), the size of each model is normalized. The object centroid is extracted directly from the model without any additional processing to the 3D model [17] and during the computation, the amount of the time consumption is smaller. In addition, this method for normalization uses global shape information to match the whole object to whole object.

\section{3D SHAPE FEATURES EXTRACTION}

Shape and size are fundamental 3D model properties providing important information about the visual appearance. In this section, we provide four categories of shape descriptors for characterizing 3D models: basic measures, size, shape indexes and moments of a distribution. These shape descriptors involved the comparison of the 3D model to its convex hull and its bounding box.

\subsection{Basic measures}

The extraction of the basic measure is the most important step to compute shape index and size descriptors. Using a combination of basic measures $M$, shape indexes $S_{I}$ can be built by defining them as algebraic combinations of the measures: $\mathrm{S}_{\mathrm{I}}=\mathrm{f}(\mathrm{M})$. Therefore, the value and the computation time of shape indexes depend on the value of the measures [14]. 3D models are represented in PSB Database test as triangle meshes and these basic measures are extracted directly from the model.

The main axis, and its length, best represents the shape and are used to compute visual concepts. To compute the main axis, we compute a reference frame for a 3D model and the inertia matrix of the point cloud and covariance matrix [18]. To compute the volume (2), polyhedron composed of each triangle mesh is obtained and the total volume of the $3 \mathrm{D}$ model is the sum of the signed volumes of all tetrahedrons [19].

$$
V=\frac{1}{6}\left(-X_{3} Y_{2} Z_{1}+X_{2} Y_{3} Z_{1}+X_{3} Y_{1} Z_{2}-X_{1} Y_{3} Z_{2}-X_{2} Y_{1} Z_{3}+X_{1} Y_{2} Z_{3}\right)
$$

The surface area of 3D model is calculated as the sum of the surfaces area of the triangles with the following formula:

$$
\text { SurfaceArea }=\frac{1}{2} \sum_{i}^{N}\left|\left(V_{i, 1}-V_{i, 0}\right) x\left(V_{i, 2}-V_{i, 0}\right)\right|
$$

With $\mathrm{V}$ is a vector containing the coordinates of the vertices of the triangle i. Other measures (e.g. Ferret diameter, Small and large radii and main plan) can be calculated from convex hull and bounding box providing the efficient comparison between two 3D models.

These measures are used in three steps: 1) as semantic concepts in ontology to define the spatial relationships, 2) to compute visual concepts and 3) as numerical descriptors to compute the 
similarity among 3D models in our content based 3D model retrieval system (CB3DMR). We consider in our ontology that each measure is the entity.

\subsection{Size}

In order to characterize the size of 3D model it is interesting to extract different size measures (diameter, length, width, height, volume and surface area) and shape indexes derived from these size measures (diameter-height ratio, diameter-diameter ratio...).

The measure equivalent spherical diameter (ESD) of an irregular shape can be used to define $3 \mathrm{D}$ model size. Let $\mathrm{SA}_{\mathrm{ch}}$ the convex hull surface area, the ESD is defined by:

$$
E S D=(4 / 3) * \Pi * \sqrt{\left(S A_{c h} / \Pi\right)^{3}}
$$

ESD measure is the diameter of a sphere of equivalent volume that gets larger or smaller as the model does. For irregular shape it is not possible to describe a 3D model using a single number that equates to the shape size. Therefore, the size $S$ of a model is defined by combining different measures $\mathrm{F}(\mathrm{S})=\{$ diameter, $\mathrm{ESD}$, volume and surface area $\}$.

\subsection{Shape indexes}

To describe the shape of 3D model, we defined a set of 3D shape indexes of each 3D model by combinations of the measures. Shape indexes tell a lot about the shape and the semantics of the 3D model and are frequently used to quantify different aspects of 3D model shape (Figure 1).

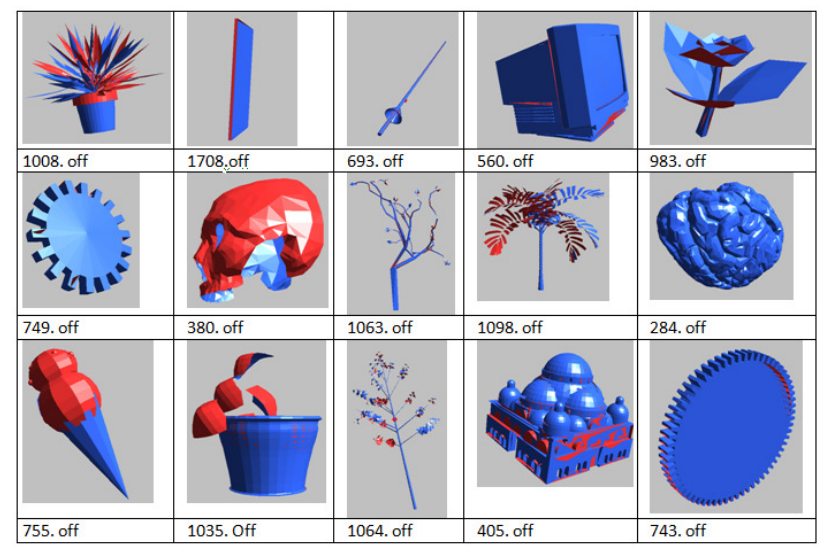

Figure 1. Samples selected from PSB Database representing different aspects

Several shape indexes have been proposed in the literature [14, 18, and 20]. In our prototypical 3D Model Search System we compute several 3D shape indexes (Table 1) characterizing the "'Sphericity," "Elongation," "Convexity," "Compactness"' of 3D model. These 3D shape indexes are computed from 3D model, convex hull and/or bounding box. Computations of these shape indexes quantified four independent properties: sphericity, Convexity, Compactness and Elongation. Each category contains several shape indexes computed by different methods. For example, to compute a sphericity shape index we can use volume and surface area, radii or main axis. To compute a compactness shape index we can also used entropy (with main axis), diameter, Surface area volume ratio or moments. However, each property can vary without affecting the other properties and their properties show that it is impossible to identify any 
shape from a single aspect [14]. Therefore, it is necessary to use different aspects of 3D shape to semantic representation and to improve the retrieval accuracy.

Table 1. Several 3D shape indexes computed.

\begin{tabular}{|l|l|l|l|l|l|l|l|l|}
\hline ID & SphericityR & SphericiteVS & Ellipticity & Circularity & Roundness & Squareness & ConvexSurf & Complexity \\
\hline 1 & 0,54267147 & 0,985590934 & 0,54267144 & 0,988374611 & 0,063894387 & 0,141580368 & 0,200955762 & 0,229430919 \\
\hline 2 & 0,67892352 & 0,971385016 & 0,6789235 & 0,985230381 & 0,04245953 & 0,184545374 & 0,211404529 & 0,14713483 \\
\hline 3 & 0,89086772 & 0,913193388 & 0,8908677 & 0,98395745 & 0,019090357 & 0,19346823 & 0,269052499 & 0,156475512 \\
\hline 4 & 0,992499913 & 0,984981198 & 0,9924999 & 0,957221798 & 0,072886105 & 0,041396828 & 0,061719714 & 0,099849517 \\
\hline 5 & 0,91332534 & 0,902407149 & 0,9133253 & 0,853145544 & 0,009782479 & 0,154164033 & 0,195353425 & 0,076064795 \\
\hline 6 & 0,90774369 & 0,954819278 & 0,9077437 & 0,927236837 & 0,018787851 & 0,187056513 & 0,219417881 & 0,10452385 \\
\hline 7 & 0,956289284 & 0,995019849 & 0,9562893 & 0,992403244 & 0,185916439 & 0,059433968 & 0,051039332 & 0,159952466 \\
\hline ID & ComplexBox & Rectangularity & ElongatAA & ElongatR & CompactECD & Concavity & Entropy & CompactVS \\
\hline 1 & 0,177309056 & 0,200955762 & 0,45732853 & 0,002195125 & 0,800054173 & 0,032341299 & 0,141735746 & 0,103187607 \\
\hline 2 & 0,139730475 & 0,211404529 & 0,32107648 & 0,003911803 & 0,761250095 & 0,019008841 & 0,14532065 & 0,082029291 \\
\hline 3 & 0,123140061 & 0,269052499 & 0,10913228 & 0,015102112 & 0,746788601 & 0,010633257 & 0,204601146 & 0,277581908 \\
\hline 4 & 0,074921354 & 0,061719714 & 0,007500087 & 0,244819579 & 0,542911769 & 0,025865605 & 0,20668771 & 0,019939768 \\
\hline 5 & 0,065544416 & 0,195353425 & 0,08667466 & 0,0194945 & 0,272626946 & 0,004625189 & 0,211576305 & 0,073316369 \\
\hline 6 & 0,097020323 & 0,219417881 & 0,09225631 & 0,018203112 & 0,42181934 & 0,01328418 & 0,211626012 & 0,064624109 \\
\hline 7 & 0,195422816 & 0,051039332 & 0,043710716 & 0,040474544 & 0,857495747 & 0,08292496 & 0,218833032 & 0,01862562 \\
\hline
\end{tabular}

The main problem is extracting $3 \mathrm{~d}$ model's shape index which represents well the aspect with good properties. In this paper, a set of shapes indexes are analyzed to select efficient one in order to combine them in categories providing different aspects to semantic annotation.

\subsection{Moments of a distribution}

Besides basic measures, size and shape indexes, moments of a distribution are also useful as descriptors to quantify the shape of the 3D model. A moment is a quantitative measure of the shape of a set of points. Mean, variance and Skewness moments are used to describe different aspects of a distribution such as how to characterize the asymmetry of distribution. To describe spatial relationships among entities, the centroid is calculated and four points from convex hull are considered (section 6.2). During this process, moments of a distribution are calculated [21] to compute numerical similarity (12 moments of the resulting four distributions).

\subsection{SHAPE INDEXES ANALYSIS}

For efficient semantic annotation and 3D models classification and retrieval, 3D models can be represented with a set of effectiveness 3D shape indexes that encode the different aspects and properties of their shapes. There are many shape indexes that can be computed from a 3D model. The main problems in this step is how to choose among a large set of shape indexes the efficient one that allows to achieve high-level performance. To solve this problem, our study concentrates on the evaluation of different shape indexes in each categories to see which one that gives the most reliable results for semantic representation and 3D model classification and retrieval. The first step to evaluate shape indexes is to study the values distribution of each shape index (Figure 2). Indeed, over the 3D shape indexes distribution shows a good separation of classes, the more 3D shape index is relevant to identify 3D models. 
International Journal of Computer Science \& Information Technology (IJCSIT) Vol 3, No 6, Dec 2011

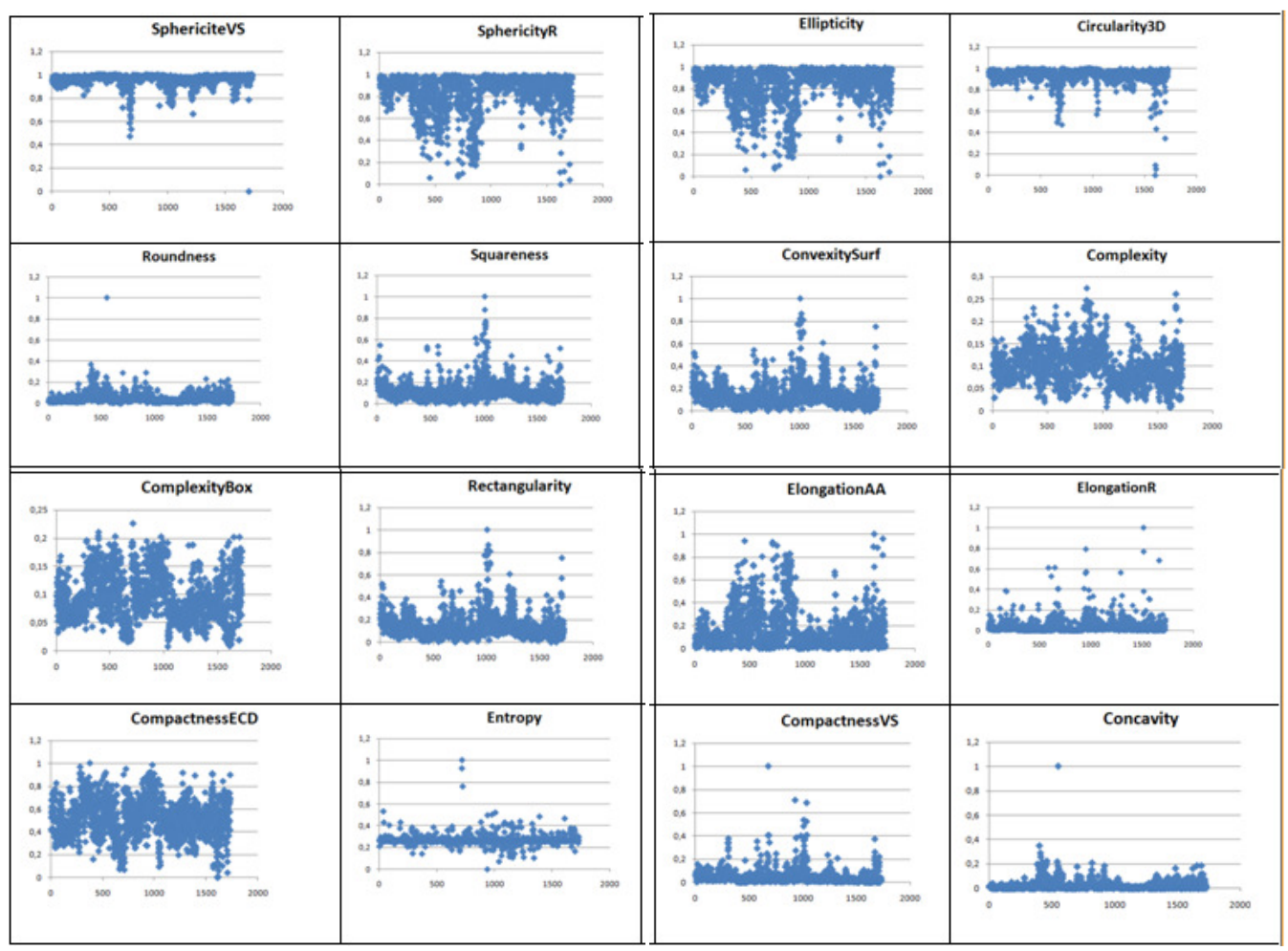

Figure 2.3D shape indexes distributions

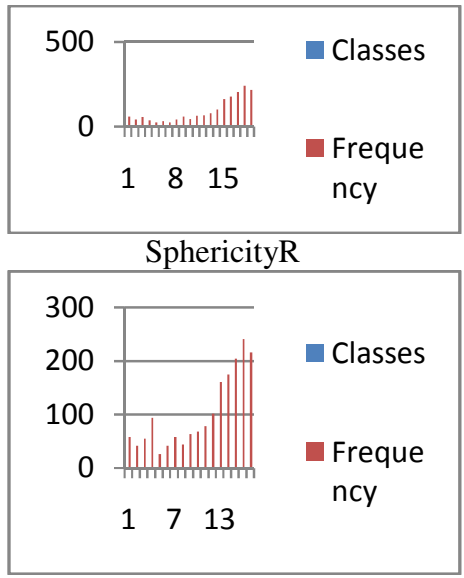

Ellipcity

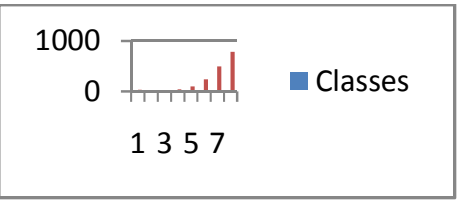

SphericityVS

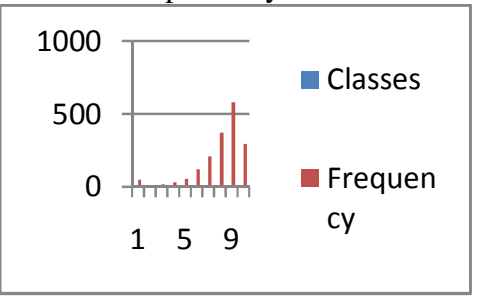

Circularity3D

Figure 3. Sphericity histogram

From distributions (Figure 2) and histogram (Figure 3), one can observes 3D shape indexes that strongly discriminate $3 \mathrm{D}$ models. The second step is to compute the weighted sum of the distances. The following formula is used to calculate the distances, which is used to determine the degree of similarity $\mathrm{S}$ between two 3D models:

$$
S=\sum_{i=1}^{n} W_{i} L_{p}(S I)_{i} \quad \sum_{i=1}^{n} W_{i}=1
$$

Where $\mathrm{W}_{\mathrm{i}}>0(\mathrm{i}=1,2 \ldots \mathrm{n})$, are the weights of $3 \mathrm{D}$ shape index $\mathrm{SI}_{\mathrm{i}}$ and $\mathrm{n}$ number of shape indexes. 
Weights are calculated and normalized during learning by k-mean algorithm using precision, recall and F-measure that allow comparing the performances of 3D shape indexes. Therefore, for each 3D shape index, we compute the average recall $(\mathrm{aR})$ and precision $(\mathrm{aP})$ on the entire 3D shape index:

$$
\begin{aligned}
& a R=\sum_{i=1}^{n} \frac{r(S I)}{n(S I)} \\
& a P=\sum_{i=1}^{n} \frac{r(S I)}{n(S I)+w(S I)}
\end{aligned}
$$

Where $\mathrm{n}(\mathrm{SI})$ is the number of models labelled by $\mathrm{SI}, \mathrm{r}(\mathrm{SI})$ is the number of models initially labelled by SI and the system has returned with the same SI, w(SI) number of the unlabeled model by the SI and found by the system with the same SI. F-measure F is the weighted harmonic mean of precision and recall. The formula of F-measure is as follows:

$$
F=2 \frac{a R a P}{a R+a P}
$$

When using average recall $(\mathrm{aR})$ and precision $(\mathrm{aP})$, it is important to specify the number of shape indexes for which at least one model was found.

To test whether the shape index was sufficient unambiguously to identify, for example, 3D model sphericity aspect, we used histogram (figure 4) to spherical 3D model examples.

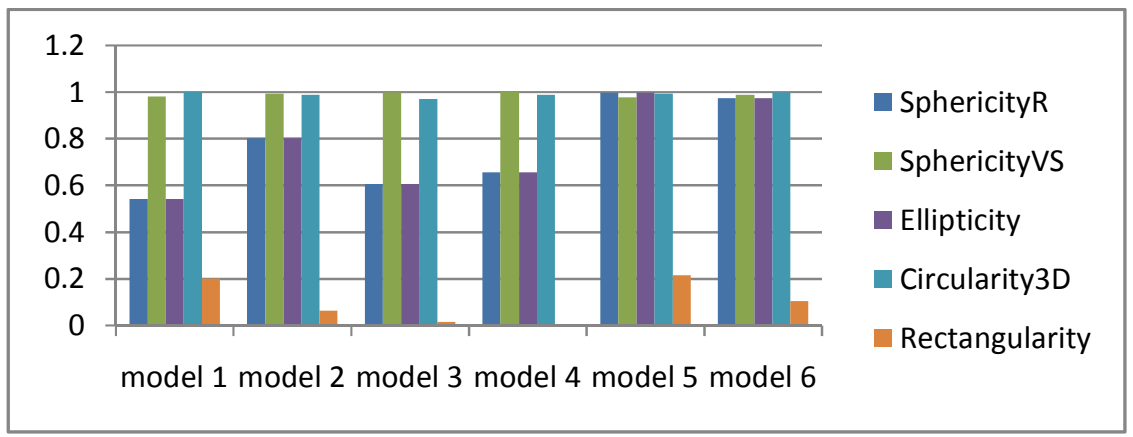

Figure 4. Sphericity histogram

\section{SHAPE INDEXES SELECTED}

3D shape indexes are selected experimentally satisfying two conditions: using precedent study to determine the best one and if its property can varies without affecting the other 3D shape indexes properties. Therefore, the 3D shape indexes selected are considered independently. We see that for Sphericity shape index selected as example in figure (Figure 5). 
International Journal of Computer Science \& Information Technology (IJCSIT) Vol 3, No 6, Dec 2011

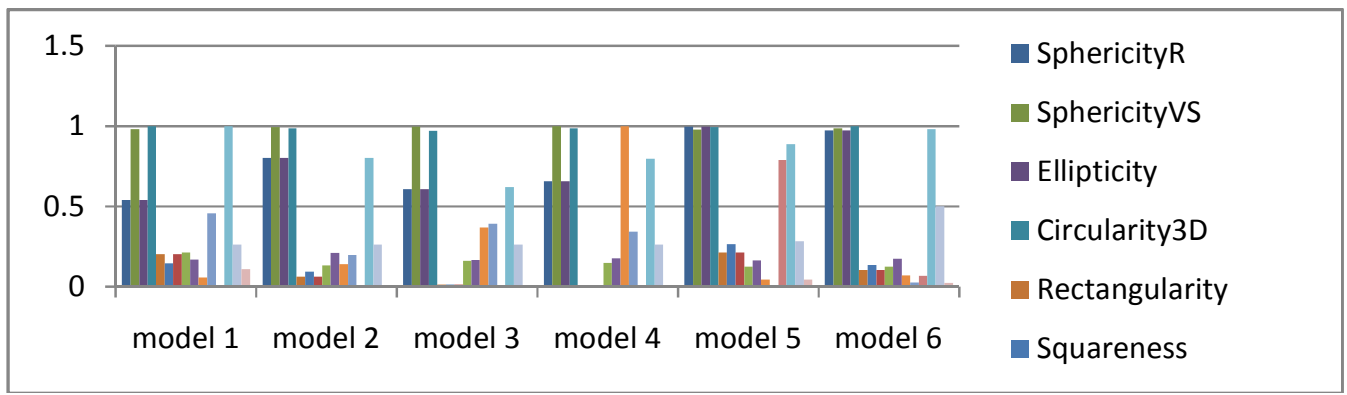

Figure 5. Shape indexes properties variations

To calculate the 3D model sphericity, we chose among several equations that use volume and surface area [20] and radii [14] as follow:

$$
\begin{gathered}
S_{1}=\frac{6 \sqrt{\pi} V}{S^{3 / 2}} \\
S_{2}=1-\frac{R_{\max }}{R_{\min }}
\end{gathered}
$$

With $\mathrm{V}$ is the volume, $\mathrm{S}$ is the surface area and $\mathrm{R}$ the rayon.

The compactness of 3D model is provided by entropy shape index calculated from main axis [18] and by volume surface area ratio:

$$
\text { Entrop }=\frac{1}{\log ^{3}} x \sum_{i=1}^{3} p i \log (p i) \quad p i=\frac{\lambda i}{\lambda 1+\lambda 2+\lambda 3} ; i=1,2,3,
$$

$\lambda$ is the object's eigenvalues along the main axes

$$
\text { Compactness }=\frac{\mathrm{V}^{2} \times 36 x \pi}{S^{3}}
$$

The convexity shape indexes are computed by comparing the volume and surface area of the convex hull to the corresponding 3D model [20]:

$$
\operatorname{Con} v_{1}=\frac{S}{S_{c h}} \quad \text { Conv } v_{2}=\frac{V}{V_{c h}}
$$

The elongation shape indexes of a 3D model are computed by comparing the lengths $\mathrm{L}_{\text {max }}, \mathrm{L}_{\text {mid }}$, and $\mathrm{L}_{\min }$ of the three edges of its bounding box.

\section{FROM SHAPE DESCRIPTORS TO SEMANTICS}

To find a concept representing the 3D model semantic information is not an easy task. The first criterion is to consider how human beings would describe objects. The system presented in this paper reduces the semantic gap including two main processes: One is automatically generating semantic concepts from 3D model shape indexes considered low-level visual features. Before this process, the problem of shape indexes is solved computing the efficient one in each category and efficient 3D shape indexes are selected experimentally (section 6). The other is spatial relationships combining directional, topological and distance relations. The system mainly use the following features : size as the more important visual feature, shape indexes to measure geometric aspects of 3D shape (e.g. sphericity, convexity,...) and spatial relationships 
among the entities to derive again high-level semantic features. High-level semantic features are derived from size and shape indexes by using machine learning techniques and from spatial relationships using ontology to improve the semantic retrieval accuracy. The K-means algorithm is employed to obtain the clustering.

\subsection{Size and Shape indexes}

To describe object size, we cluster volume, surface area, diameter and ESD to construct the following visual terms [15] that are commonly used: Long, big, small, short, large, wide, high, thick and narrow. The shape of object has many meanings and shape indexes provide a set of terms commonly used to describe visually a set of objects using linguistic terms[15] like: round, angular, rectangular, symmetric, smooth, irregular, convex,... . Each shape index gives a set of visual concepts [22]. Table 2 shows an example of sphericity Concepts and Class Limits.

Table 2. Sphericity and Roundness Concepts in Class Limits

\begin{tabular}{|l|c|c|}
\hline sphericity Concepts & Class Limits & Geometric Mid-Point \\
\hline Very high sphericity & $0.894-0.949$ & 0.949 \\
\hline High sphericity & $0.775-0.894$ & 0.837 \\
\hline Moderate sphericity & $0.632-0.775$ & 0.707 \\
\hline Low sphericity & $0.447-0.632$ & 0.548 \\
\hline Very low sphericity & $0.000-0.447$ & 0.316 \\
\hline Roundness Concepts & Class Limits & Geometric Mid-Point \\
\hline Angular & $0-0.125$ & 0.09 \\
\hline Sub- angular & $0.125-0.250$ & 0.18 \\
\hline Sub-rounded & $0.250-0.500$ & 0.35 \\
\hline Rounded & $0.500-1$ & 0.71 \\
\hline
\end{tabular}

The number of visual concepts is defined by fixing the parameter $\mathrm{K}$ using k-mean algorithm in evaluation shape indexes step experimentally. The four categories representing different aspects are used in content based 3D model retrieval numerically to identify models.
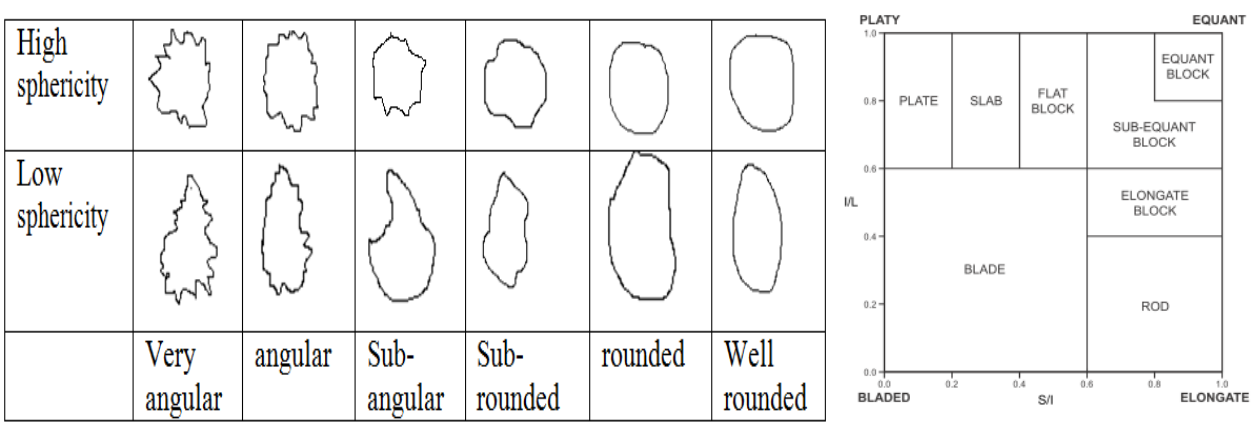

Figure 6. Other concepts to describe models

To increase the knowledge base, all other shape indexes are directly added (e.g. rectangular, symmetric, smooth, irregular) giving each one a set of visual concepts or combining them to include other concepts (Figure 6). Combining shape indexes, we can describe a 3D model by 
several other concepts (e.g. discoid, very oblate spheroid, oblate spheroid blade). In this context, we exploit a diagram introduced by T. Zingg in 1935 [22] to plot the relative dimensions of the long, short, and intermediate axes of an object (Figure 6). Therefore, a set of new visual concepts are included.

\subsection{Spatial Relationships}

Shape indexes describing geometrical aspects of 3D shapes are not sufficient to represent the semantic information of $3 \mathrm{D}$ model ignoring the spatial relationships. The second process to reduce the semantic gap is to use the spatial relationship representing directional, topological and distance relationships to derive high-level semantic features. Therefore, four points from convex hull are considered: centroid (P1), the closest point to centroid (P2), the farthest point to centroid (P3), and the farthest point to $\mathrm{P} 3$ (P4). In the first step, the center of gravity is located. Then, we calculate other points using Euclidean distance.

From these points, we can compute polygons [23], lines, plans and angles. This set of entities allows the description of model independently of their size, rotation, translation or line type [23]. Angles allow computing various directions and to describe the directional relationships using the following concepts: Left, Right, Up, Down or Touch, and Front to describe topological relationships. The distances can be computed from a point to point, line to line, point to line, point to plan and line to plan. To describe the distance relationship between two entities, usually the following concepts are used: very near, near, far, far away.

Based on the spatial relationships and their properties, we build the ontology-based 3D model retrieval using the web ontology language (OWL) [24].

\section{ONTOLOGY BASED 3D MODEL RETRIEVAL}

\subsection{Ontology}

Low-level descriptors for the measure, size, shape and spatial relationships of each 3D model are extracted. These descriptors are associated with high-level concepts to reduce the semantic gap and to form a vocabulary structured in ontology. This ontology, independent of the application domain, is used as a guide for describing the models of a domain of expertise, as in [24] since experts often use and share a generic visual vocabulary to describe objects of their domain [15]. Our ontology is structured in two parts: 1) the first part is content based ontology containing numerical data for interpreting 3D model content. In this paper, the query by example is adopted to query the ontology. This query is reformulated to exploit SPARQL engine, then, allows the user to query Database using semantic concepts and provides a numerical similarity among models. 2) Concerning the second part, used for semantic based 3D Model retrieval, the goal is to encode generic visual concepts used by humans to describe real world objects and to develop a rich set of 3D model semantic representation for interpreting its semantic content. Therefore, an Ontology-Based 3D Model Retrieval independent of the application domain is proposed.

\subsection{Reasoning}

To augment the knowledge base with new concepts, a combination of semantic concepts is often required. The ontology facilitates the inference the spatial relation; therefore, other semantic property can be inferred by rule engine. The implicit rules are defined using OWL properties such as similarity owl: SameAs.

$<$ RDF:Description rdf:about="\#sphericity" $>$

$<$ owl:sameAs rdf: resource="\#3Dcircularity "/>

$</$ Rdf: Description $>$ 
We can define other explicit rules to infer spatial relationships based on other relationships. For example, if a 3D model is nearly circular but not compact it must be ragged or star-like. Another example, if a 3D model is angular and has rounded edges then it is subangular (section 7).

\subsection{Query by semantic concepts}

In content based 3D model retrieval, a 3D model is directly chosen as a query in the database. As explained in section 8.1, Visual concepts are used for annotation with Ontology-Based 3D Model Retrieval purpose. Our approach combines query by example with the query by semantic concepts QBSC for semantic 3D model indexing and retrieval purposes transforming a query 3D model into a set of visual concepts. The QBSC retrieval system combines 3D models database $M=\left\{M_{1}, \ldots, M_{D}\right\}$ and a vocabulary $V=\left\{1_{1}, \ldots, 1_{v}\right\}$ of semantic labels . All database $3 \mathrm{D}$ models are annotated with a label composed of words from $\mathrm{V}$. The elements composing annotations are structured using the logical operators \{or, and $\}$ ) to query a database. The result of retrieval is the sub-set of the indexed 3D models with associated annotations and numerical values. Therefore, retrieval can be based on the semantic description of the query 3D model. For example, the query "Sphericity $=$ high and Roundness $=$ angular" retrieves the 3D models annotated as containing angular with highSphericity.

SPARQL engine admits the use of numeric values to compute the similarity on the retrieved models that are semantically similar. The query can easily be adapted to obtain the distance between any pair of 3D models. Therefore, the similarity between two models in this step is measured using a distance between their 3D shape indexes, measures, size, and moments of a distribution.

\section{EXPERIMENTAL RESUltS}

In this section, we evaluate content and semantic approaches for improving performance of 3D model retrieval in our (SB3DMR) system. To evaluate the content approach, first, several shape indexes are compared to select the best one as the feature vector in both the machine learning algorithm (section 5) and the (CB3DMR) system. Second, shape indexes selected (SID), measures (MD), moments of distribution (MDD), spatial descriptors (SD) and the combinations of them (SIMMSD) are also evaluated comparing the retrieval results of the single descriptors and combined descriptors. We tested our descriptors on the Princeton Shape Benchmark Database (PSB) [28] using L1 (Manhattan) as similarity measure.

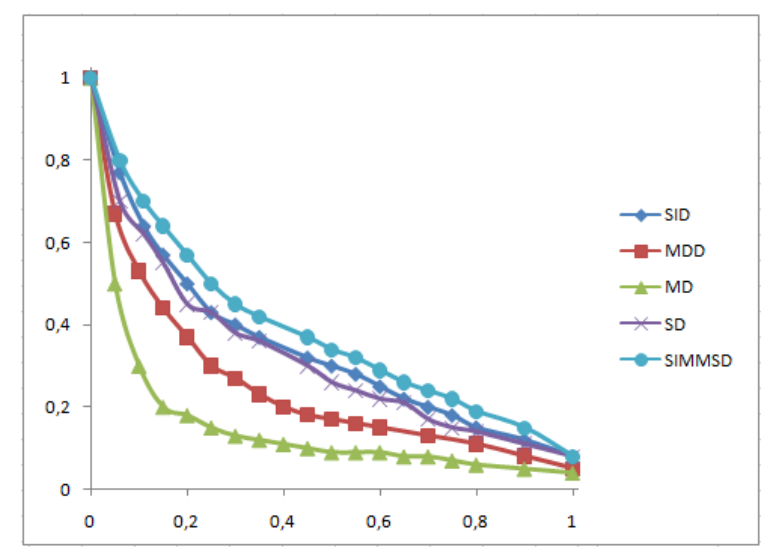

Figure 7: The precision-recall curves of our descriptors 
International Journal of Computer Science \& Information Technology (IJCSIT) Vol 3, No 6, Dec 2011

As shown in figure 7, and for the single shape descriptors, shape index and spatial relationship are the best among all the single shape descriptors. But, the combination of all descriptors performs better and it is effective in improving retrieval performance.

The average times that are used to compute all shape descriptors is 0,114 seconds for a model, using the Princeton Shape Benchmark Database (Table 3).

Table 3. Time to compute all descriptors

\begin{tabular}{|c|c|c|c|c|}
\hline Example model & $\begin{array}{c}\text { Number of } \\
\text { edges }\end{array}$ & $\begin{array}{c}\text { number of } \\
\text { polygons }\end{array}$ & $\begin{array}{c}\text { Number of } \\
\text { vertices }\end{array}$ & Time \\
\hline 1 & 1638 & 546 & 341 & 0,128405697 \\
\hline 2 & 648 & 228 & 100 & 0,131520481 \\
\hline 3 & 1476 & 492 & 298 & 0,085323701 \\
\hline 4 & 1224 & 408 & 216 & 0,234916242 \\
\hline 5 & 40626 & 13542 & 7056 & 0,80845978 \\
\hline
\end{tabular}

Query by example approach (QBE) is used to query Database and we can directly retrieval models as shown in Figure 8 and 9. The 12 most similar models are extracting and return to do a user by $2 \mathrm{D}$ images. To visualize the $3 \mathrm{D}$ models in the $3 \mathrm{D}$ space, the user clicks the button or image.

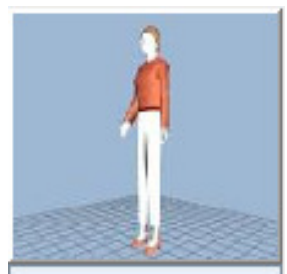

Figure 8. query model

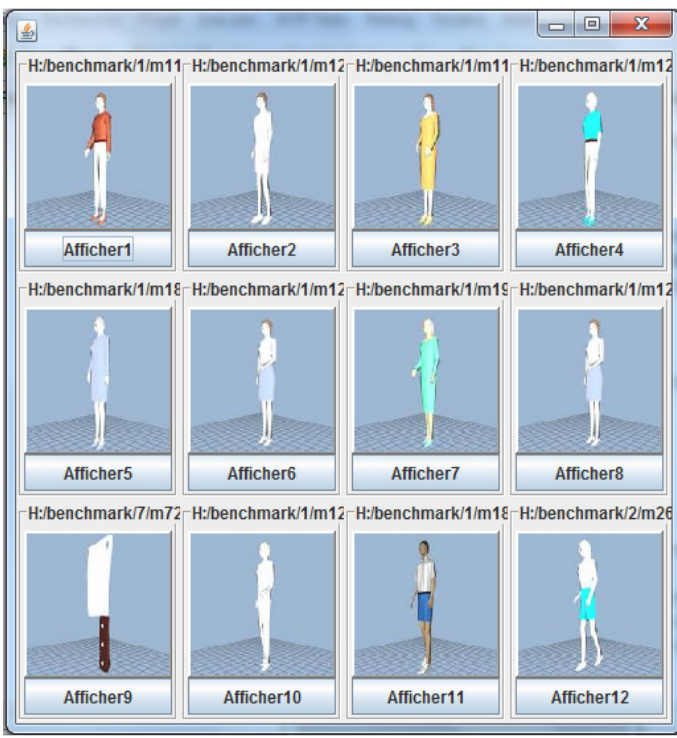

SID

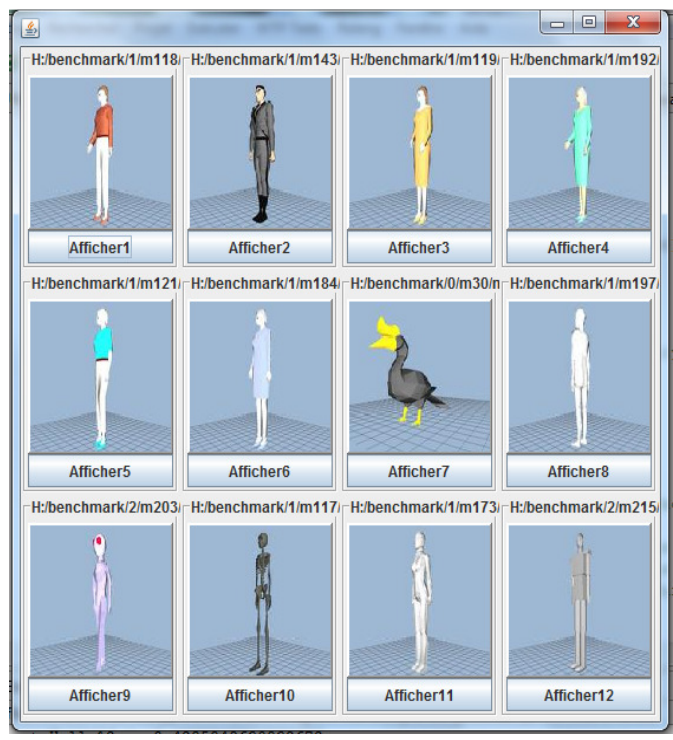

SD 


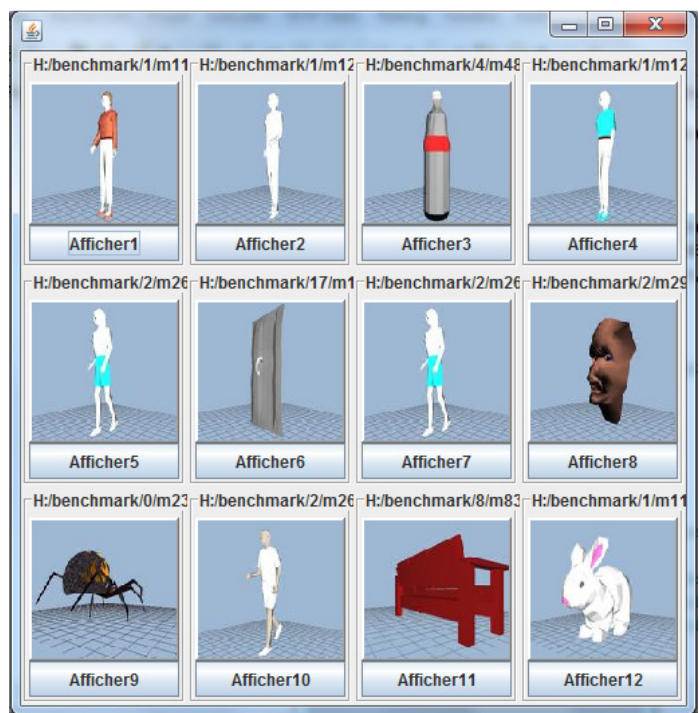

MD

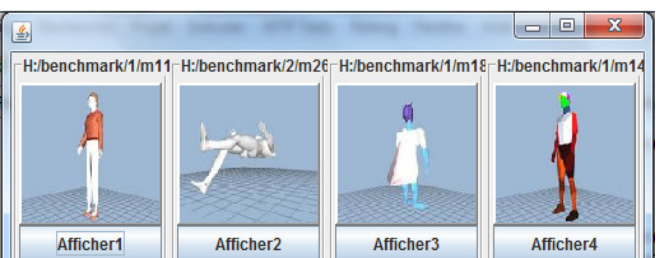

H:benchmark/1/m18 H:lbenchmark/14/m1 H:lbenchmark/11/m1 H:/benchmark/1/m1

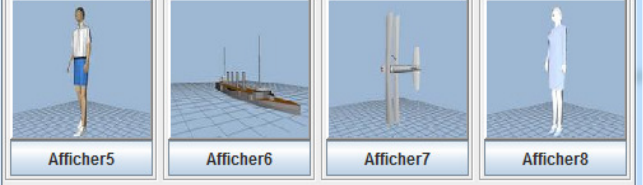

H:benchmark/11/m1 - H:lbenchmark/1/m12 H:/benchmark/1/m12 - H:lbenchmark/11/m

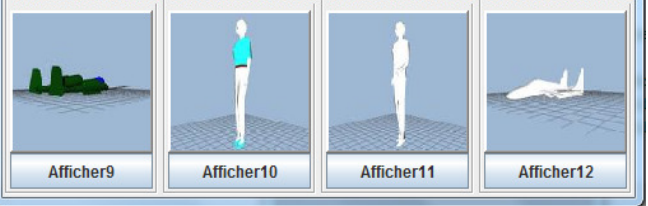

MDD

Figure 9. Models found examples for different descriptors

We also compared our combined descriptor (SIMMSD) to four existing methods: shape distribution (D2) [26], volume area ratio [19], 3D Harmonics [27] and 3D Moments descriptor. The figure 10 shows the Recall and Precision graphs for each method that our proposed descriptor performs well.

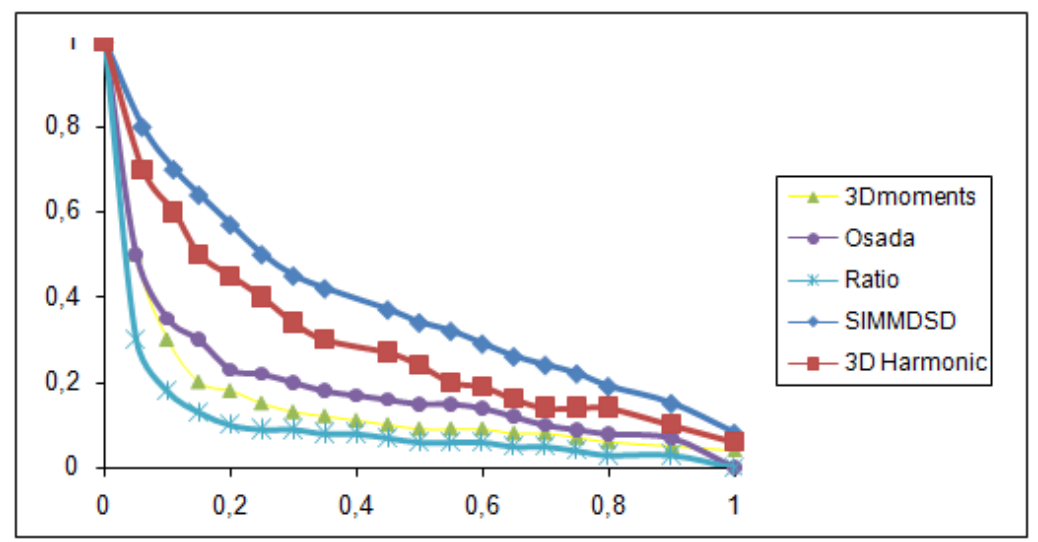

Figure 10: The precision-recall curves of our descriptor compared with other methods

In the second stage, semantic-based 3D model retrieval system (SB3DMR) is used to evaluate our semantic approach. QBSC is used to query knowledge base transforming a query 3D model (QBE) into a set of visual concepts. The result is the sub-set of the 3D models semantically selected exploiting SPARQL engine. In our system, size, shape index and spatial descriptors are associated with high-level semantic concepts and measures are considered entities to compute relationships. Other shape index concepts are directly added (e.g. Rectangularity, Ellipticity...) or inferred using rule engine. Moments of distribution descriptors are only used in CB3DMR system and Color descriptors are not implemented neither the texture descriptors in both approaches CB3DMR and SB3DMR. 


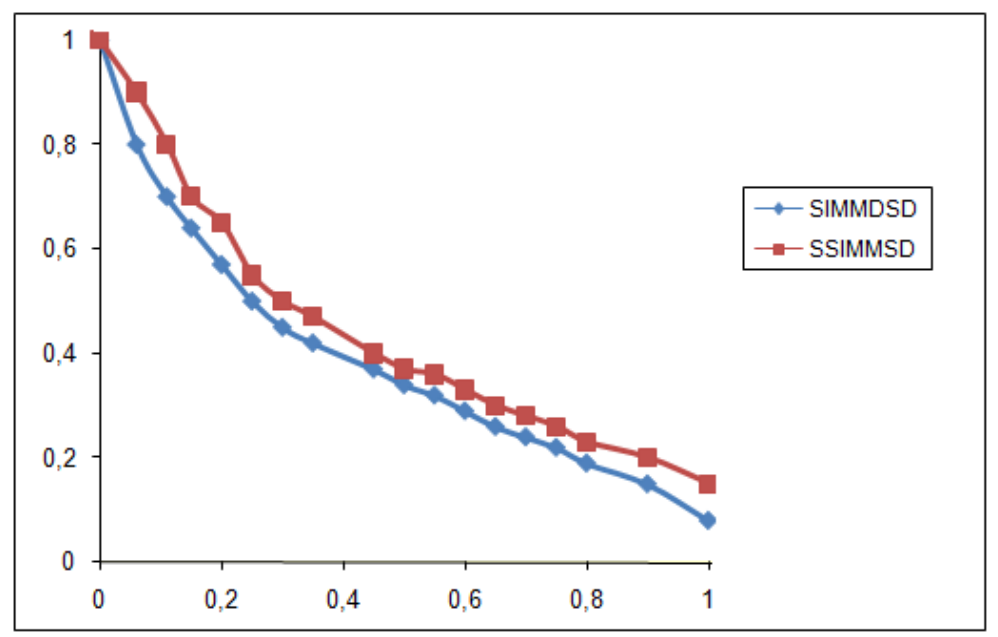

Figure 11: The precision-recall curves to compare our descriptors

The Recall and Precision is used also to evaluate the performance of semantic approach (SSIMMSD) compared to our content approach (Figure 11). The proposed approach performance is linked to the semantic gap reduced in two steps: In the first one using efficient shape indexes to annotate 3D models and in the second one using QBSC to query knowledge base structured in ontology containing a number of concepts analyzed and integrated directly or inferred using rule engine.

\section{CONCLUSION}

A new approach for semantic based 3D model retrieval is introduced in this paper. First, invariant descriptors are extracted and analyzed to efficient semantic annotation and to improve the retrieval accuracy. Shape descriptors selected are used as feature vector in machine learning to label 3D model and semantic concepts are structured in ontology to infer other concepts. Second, spatial relationships are extracted to increase the knowledge base and the QBSC is used to reduce the semantic gap exploiting SPARQL engine to retrieve 3D model. The new approach is tested with a large 3D database using the SB3DMR system developed, which allows us to show the relevance of our method. To improve our system, it is interesting to increase our knowledge base by other concepts to 3D model interpreting. Soon, the ontology will be enriched with color concepts.

\section{REFERENCES}

[1] Tangelder, J.W.H. \& Veltkamp, R.C.(2008) "A survey of content based 3D shape retrieval methods" in: Multimedia Tools and Applications Vol. 39 Issue 3

[2] Tangelder, J.W.H. \& Veltkamp, R.C.(2004) “A survey of content based 3D shape retrieval methods" in: Shape Modeling Applications. Proceedings

[3] P. Xiang, Z. San-Yuan, \& Y. Xiu-Zi (2009) "A Survey of Content-Based 3D Model Retrieval with Semantic Features" in Chinese Journal of Computers Vol. 32, N 6

[4] R. Ohbuchi \& T. Shimizu, (2008) "Ranking on semantic manifold for shape-based 3d model retrieval", in Proc. Multimedia Information Retrieval, pp.411-418.

[5] G. Leifman, R. Meir \& A. Tal(2005) "Semantic-oriented 3d shape retrieval using relevance feedback" The Visual Computer .Vol. 21, No 8-10, 865-875 
International Journal of Computer Science \& Information Technology (IJCSIT) Vol 3, No 6, Dec 2011

[6] S. Goodall, P.H. Lewis, \& K. Martinez, (2005) "Towards Automatic Classification of 3-D Museum Artifacts Using Ontological Concepts", in Proc. CIVR, pp.435-444.

[7] Akgul, C. B., Sankur, B., Yemez, Y., \& Schmitt, F. (2008). “ Similarity score fusion by ranking risk minimization for $3 d$ object retrieval". In Eurographics workshop on 3D object retrieval Crete, Greece

[8] B. Gao, H. Zheng \& S. Zhang (2009) “Ontology-based design information extraction and retrieval”. In: Artificial Intelligence and Computational Intelligence. AICI '09.

[9] JL Shih, \&H. Chen (2008) "3D Shape Retrieval Integrated with Classification Information" in Multimedia Tools and Applications Vol. 43, Issue: 1, 45-62

[10] S. Hou, K. Lou \& K. Ramani, (2005) "SVM-based Semantic Clustering and Retrieval of a 3D Model Database" in CAD journal 2, 155--164.

[11] X. Wang, T. Lv, S. Wang, \& Z. Wang (2008) "An Ontology and SWRL Based 3D Model Retrieval System". AIRS : 335-344.

[12] P. Marios \& C. Chiara (2009) “A semantic based framework for managing, searching and retrieving $3 D$ resources" in VSMM Conference Program.

[13] S. Arasan, A.S. Hasiloglu , \& S. Akbulut (2010) " Shape Properties of Natural and Crushed Aggregate using Image Analysis" in IJCSER JOURNAL Vol. 1, No 2,

[14] G. Thibault, Fertil B., J. Sequeira \& Mari J-L. (2010) "Shape and texture indexes. Application to cell nuclei classification” MajecSTIC, vol. 15, no 2 (117p.) 73-97

[15] C. Hudelot (2005) "Towards a Cognitive Vision Platform for Semantic Image Interpretation; Application to the Recognition of Biological Organisms" thesis Nice Sophia-Antipolis University

[16] C. Zhang \& T. Chen (2001) "Indexing and retrieval of 3D models aided by active learning", in Proc. ACM Multimedia, pp.615-616.

[17] Kim, A., Song, J., Gwun, O., \& Shin, S. (2008) “3D Model Retrieval Using Convex Hull and Curvature" In Proceedings of CGVR, 136-141.

[18] Olivier T., Fabrice D., Clare H., Vincent B. \& Patrick L. (2006) “A quantitative approach to the study of cell shapes and interactions during early chordate embryogenesis" Current Biology 16, 345-358.

[19] Cha Z. \& Tsuhan C. (2001) "Efficient feature extraction for 2D/3D objects in mesh representation”. In IEEE International Conference on Image Processing (ICIP 2001), pages 935938, Thessaloniki, Greece,

[20] A. C. Silva, V. R. da Silva Junior, A. de Almeida Neto, \& A. C. de Paiva: (2005) "Diagnosis of Lung Nodule Using Reinforcement Learning and Geometric Measures". MLDM: Leipzig, Germany 295-304

[21] Pedro J.Ballester \& W. Graham Richards (2007) “Ultrafast Shape Recognition to Search Compound Databases for Similar Molecular Shapes" in Wiley InterScience

[22] Blott, S.J. \& Pye, K. (2008) "Particle shape: a review and new methods of characterization and classification”. Sedimentology 55, 31-63

[23] FONSECA Manuel J. , FERREIRA Alfredo \&JORGE Joaquim A. (2005) "Generic shape classification for retrieval" International Workshop on Graphics Recognition No6, Hong Kong , CHINE

[24] N. Maillot, Monique T. \& Alain B. (2003) "A Visual Concept Ontology for Automatic Image Recognition" in 2nd ISWC Conference, Sanibel Island (FL, USA).

[25] Mezaris, V., Kompatsiaris \& Strintzis, M.G. (2004) "Region-Based Image Retrieval Using an Object Ontology and Relevance Feedback”. In Proceedings of EURASIP J. Adv. Sig. Proc., 886-901. 
International Journal of Computer Science \& Information Technology (IJCSIT) Vol 3, No 6, Dec 2011

[26] R. Osada, T. Funkhouser, B. Chazelle \& D. Dobkin, (2002 ) "Shape distributions", ACM Transactions on Graphics, vol. 21, no. 4, pp. 807-832.

[27] M. Kazhdan \& T. Funkhouser (2002) "Harmonic 3D shape matching”, ACM SIGGRAPH Vol. 12, Issue: 212, Pages: 191

[28] P. Shilane, P.Min,M. Kazhdan, \& T. Funkhouser. (2004) "The princeton shape benchmark". In Shape Modèleing International .

[29] B. GAO, S. ZHANG \& X. PAN “Semantic-Oriented 3D Model classification and Retrieval Using Gaussian Processes” in Journal of Computational Information Systems 7:4 2011.

[30] H. Laga "3D Shape Classification and Retrieval Using Heterogenous Features and Supervised Learning” InTech, January 2009.

[31] C. B. Akgul, B. Sankur, Y. Yemez \& F. Schmitt. "Similarity Learning for 3D Object Retrieval using Relevance Feedback and Risk Minimization". Int. Journal of Computer Vision, Special Issue on 3D Object Retrieval, vol. 89, no. 2-3, pp. 392-407, 2010

[32] B. Leng, Z. Qin, \& L. Li (2007) "Support Vector Machine active learning for 3D model retrieval" Journal of Zhejiang University SCIENCE Vol: 8, Issue: 12,

[33] R. Albertoni, L. Papaleo, M. Pitikakis, F. Robbiano, M. Spagnuolo \& G. Vasilakis, (2005) "Ontology-Based Searching Framework for Digital Shapes", in Proc. OTM Workshops , pp.896-905.

\section{Authors}

My abdellah Kassimi is a $\mathrm{PhD}$ student at Sidi Med Ben Abdellah University (GRMS2I group) in Morocco. He received his DESS in Computer Science from the University of Sidi Md Ben Abdellah in 2007. His current research interests are 3D indexing and retrieval, 3D shape indexes, semantic and ontology.

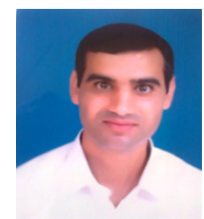

Omar El Beqqali is currently Professor at Sidi Med Ben Abdellah University. He is holding a Master in Computer Sciences and a PhD respectively from INSA-Lyon and Claude Bernard University in France. $\mathrm{He}$ is leading the 'GRMS2I' research group since 2005 (Information Systems engineering and modeling) of USMBA and the Research-Training PhD Unit 'SM3I'. His main interests include Supply Chain field, distributed databases and Pervasive information Systems. He also participated to MED-IST project meetings. O. El Beqqali was visiting professor at UCB-Lyon1 University, INSA-Lyon, Lyon2 University and UIC (University of Illinois of Chicago). He is also an editorial board member of the International Journal of Product Lifecycle Management (IJPLM). 